\title{
XGPON statistical multiplexing analysis with heterogeneous users
}

\author{
Anjali Gupta ${ }^{1}$, Carmen Mas Machuca ${ }^{2}$, Senior Member, IEEE and Abhishek Dixit ${ }^{1}$ \\ ${ }^{1}$ Indian Institute of Technology (IIT), Delhi \\ ${ }^{2}$ Technical University of Munich (TUM), Germany \\ Tel: +91-9779895645, e-mail: 79anjaligupta@gmail.com
}

\begin{abstract}
Optical access networks are rapidly evolving mainly driven by the higher bandwidth requirements. XGPON is one of the most cost-effective solutions to upgrade existing GPON since it reuses existing optical distribution networks. Thanks to the high XGPON capacity, these networks can connect heterogeneous users such as base stations (BS), residential users (RU) or business users (BU). Statistical multiplexing of XGPON allows assigning different bandwidth to the different users based on their demands and always guaranteeing the agreed service level agreements. This work aims at evaluating the impact of packet delay and throughput for different XGPON scenarios for different user distributions. Furthermore, the paper evaluates the flexibility to cope with extra traffic caused by existing protection schemes.
\end{abstract}

Keywords: XGPON, DBA, network convergence, protection, throughput, packet delay

\section{INTRODUCTION}

Passive optical network (PON) is optical access network that can provide high data rates to its end users. A PON, having a tree topology, interconnects a single optical line terminal (OLT) to multiple optical network units (ONU) via a passive splitter. All ONUs share the feeder fiber interconnecting the OLT and the splitter. Hence, multiplexing techniques like time-, wavelength-, and code- division multiplexing (TDM, WDM, CDM respectively) or any combination can be used. This work focuses on TDM PONs because of their extensive deployment and advantages, e.g., standardization, use of the same wavelength by all users. In TDM PONs, the OLT broadcasts messages in downstream (DS) to all ONUs and each ONU gets upstream (US) opportunity granted by OLT to avoid collisions. First gigabit-capable PON (GPON) ITU-T recs. [1] aims at providing aggregated $2.5 \mathrm{Gbps}$ line rates. However, due to an exponential increase in data demands, the next generation 10-gigabit-capable PON (XGPON) [2] has been proposed, which can provide line rates of $10 \mathrm{Gbps}$ at affordable migration costs, thanks to the reuse of the GPON distribution network (i.e., same fiber and power splitter). The high data rates offered by XGPON support network convergence, i.e., connecting multiple types of users with different bandwidth and availability requirements (e.g., base stations (BS), business users (BU), and residential users (RU)). Since each user uses its network capacity at different times of the day, connecting heterogeneous users can provide a better user experience and quality of service (QoS) by allowing users with higher demands to utilize the left-over capacity. Furthermore, connection availability varies based on the user type. There are several protection schemes proposed in the literature [3], which can be applied to users requiring higher availability like BS.

ITU-T G.987.3 [4] recommendation proposes a reference model for dynamic bandwidth allocation (DBA) which defines bandwidth fairness during resource allocation, but it is left on the service provider to choose the best algorithm for achieving the same. It also explains the provision of incoming traffic segregation on the basis of different traffic classes to maintain QoS requirements. Several DBA mechanisms like GIANT [5], IACG [6], EBU [7], IBU [8], FEx-DBA [9] demonstrates the delay performance of the network for different traffic classes. In these allocation mechanisms, higher priority traffic classes enjoy better throughput and delay performance because of relaxed QoS requirements or by degrading the performance of lower priority traffic classes. Technology advancements like IoT, M2M (Machine to Machine), cloud computing, VR (Virtual Reality), AR (Augmented Reality) have more critical requirements in terms of delay, security, etc. depending on the services. All DBA mechanisms discussed above, either face over-reporting problem or require complicated programming demanding lots of memory and processing time. A. Dixit et al. [10] propose a simple and effective reporting method for LR-EPON to deal with such problem and we are implementing same technique in XGPONs to deal with the issue of over-reporting. We are analyzing our system by examining average packet delays and throughput for heterogeneous users by providing priority to the customers based on their network availability demands. Moreover, we are studying the impact on network performance in case of failure of a protected network, because of extra traffic coming from ONU of the failed protected network.

In Section 2, we are discussing our DBA implementation and simulation parameters. Section 3 contains the results and analysis performed on the network. Finally, we are concluding the paper in Section 4.

\section{Dynamic bandwidth allocation}

Transmission convergence (TC) layer is equivalent to data link layer of OSI model, i.e., it handles all the functionalities of GPONs like media access control (MAC), DBA, encapsulation, synchronization, etc. The 
MAC states the way of accessing the channel in US, US BW assignment, etc. by considering the different delays between each ONU and the OLT. The MAC equalizes all OLT-ONU delays such that all ONUs are logically equidistant from the OLT. The OLT sends grant messages to ONUs in the form of BWmap field of the TC layer, which defines the start and the end time of the data burst forwarded by each ONU. The algorithm by which OLT calculate the US burst time slot assigned to each ONU is called DBA algorithm.

\subsection{XGPON DBA}

The bandwidth fairness reference model mentioned in [4] forms the basis of XGPON DBA. In our algorithm, OLT keeps track of queue statuses of every ONU based on their US dynamic bandwidth report $(D B R u)$, which contains the value of outstanding traffic at respective ONUs. The $D B R u$ field strictly carries the number of unreported packets (in bytes) instead of total buffer occupancy value. OLT sends grants to ONUs based on their queue statuses. It does not grant any BW once the ONU's buffer gets empty but ONU can start getting traffic again. So, OLT grants a minimum BW of 36 bytes after every 10 DS $(1.25 \mathrm{~ms})$ frames for such ONUs for reporting their queue statuses. OLT calculates a specific guaranteed bandwidth $(R g)$ value for each ONU such that, the sum of $R g$ value for all ONUs is equal to channel capacity. An ONU can get a BW more than $R g$ in case the instantaneous network load is low (availability of surplus BW, where surplus BW is the BW left after assignment up to $R g$ to the ONUs based on their demands) and the request from the ONU is higher than $R g$. In case we are defining priority, it always refers to the preference given during division of surplus BW. We are using priority in the context of handling extra traffic coming from an ONU of failed protected network and also on the basis of network availability requirements of different users. A user with the highest priority can get whole surplus BW if its demand is high, a user with lower priority can get a share of surplus bandwidth only if the requirement of higher priority users is satisfied. DBA process can be explained by an example: let us suppose there are three users $\mathrm{u} 1, \mathrm{u} 2, \mathrm{u} 3$ with $\mathrm{u} 1$ having the highest priority and $\mathrm{u} 3$ having the lowest priority. Let the channel capacity for one US frame (in bytes) is 1200 bytes. We are defining weights as w1:w2:w3 based on different type of user for guaranteed BW calculations. Let us suppose these weights are 2:1:1 in our example and so $R g$ for u1, u2 and u3 is $600,300,300$ bytes respectively. So, if demand is $200,800,700$ bytes from u1, u2 and u3; assigned BW is 200, 700, 300 bytes, and similarly if the request is $900,100,500$ bytes; allocated BW is 800 , 100,300 bytes, and so on.

\subsection{Simulation Parameters}

For our OMNeT++ implementation, we are simulating a network having a maximum split ratio of 1:32 and US line rates of $10 \mathrm{Gbps}$, of XGS-PON [11] (symmetric) network where we are placing ONUs at random distances of 10-20 kms from OLT. A self-similar traffic model with a Hurst parameter of 0.8 and shape parameter values of 1.4 and 1.2 for ON, OFF periods respectively are used to model traffic of ONUs, as discussed in detail in [12]. We are performing packet fragmentation according to [4] and using fixed size XGEM frames of 1526 bytes. We are considering all other recommended parameters of XGPON for our simulations, like fixed frame sizes of 125 $\mu \mathrm{s}$, the response time of $36 \mu \mathrm{s}$, guard bands, etc. For every simulation, we fixed the simulation time to $300 \mathrm{~s}$.

To check the reliability of our algorithm, we are initially simulating our network for homogenous users (32 users with the same priority, 500 Mbps mean peak rates for Pareto traffic and w1:w2:w3 as 1:1:1). The obtained simulation results are aligning with theoretical results based on queueing theory [13]. Then we are simulating the network using same parameters but assigning different priorities to the users for studying the impact of priority assignment and results are displayed in Fig. 1. It is clear that the highest priority traffic outperforms the case with all users having the same priority, but lower priority traffics are facing penalty for this improved performance.

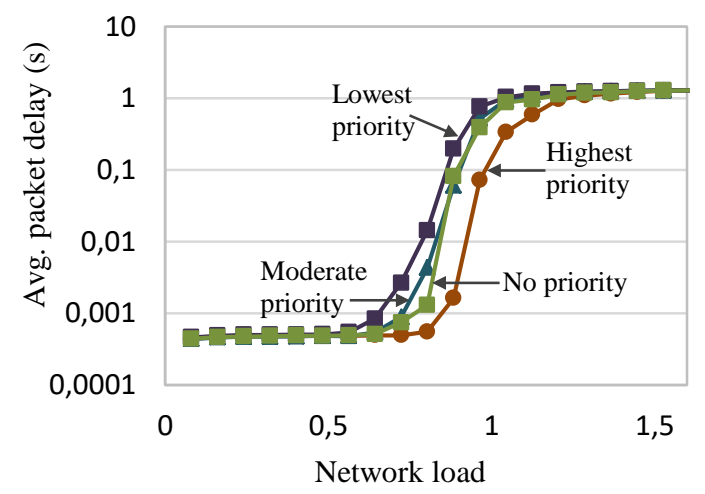

Fig 1. A comparison of average packet delays for a network having homogeneous users with users assigned different priorities (hence three curves based on three priorities) to the same network with all users having the same priority (denoted by no priority traffic). 


\section{Case study for heterogeneous users}

We are assigning weights as 30:10:1 for BS:BU:RU in our case study. Besides $R g$, we are allocating peak rates and buffer sizes according to this factor. We are using buffer sizes of $10 \mathrm{MB}$ and mean peak rate of $100 \mathrm{Mbps}$ for RUs. We are providing highest priority to BSs, then to BU and the lowest priority to RUs. The reason for this priority assignment is that the BSs requires higher availability and more bandwidth than BUs, and this requirement is higher for BUs than RUs. Moreover, BSs are responsible for carrying protection traffic from BS of another failed access network (e.g., using the $\mu$ Wave protection [3]). Since the considered power splitter is 1:32, the number of heterogeneity cases are limited to 14 cases as the sum of $R g$ of all ONUs should not exceed the channel capacity (i.e., $10 \mathrm{Gbps}$ ). The particular case of $2 \mathrm{BSs}, 9 \mathrm{BUs}$, and 10 RUs is considered for detailed analysis and all the results for heterogeneous scenario correspond to this case.

In the presence of protection mechanism, BS of the supporting network has two queues. The second queue is used to store the traffic coming from BS of the failed node. Due to the connection availability of unprotected optical access networks ( 3 nines), no $R g$ to the traffic coming from a failed node is considered (i.e., the planning is done assuming a working state of all access networks). In case of a failure, the protected traffic can pass through the supporting network only if surplus BW is available, getting allocated BW according to a BS priority.

\subsection{Throughput evaluation}

Throughput is calculated as the number of packets passing successfully through the network per unit time, which can be expressed as (packets_sent $\left(t_{2}\right)$ - packets_sent $\left.\left(t_{1}\right)\right) /\left(t_{2}-t_{1}\right)$ for $t_{2}>t_{1}$.

For the network working under normal conditions (i.e., with no protection traffic coming from a failed network), the throughput curves are depicted in Fig. 2a. The throughput curves saturate to corresponding $R g$. It can be observed, that $R g$ is reached earliest by BS, and even overpasses it due to the highest assigned priority and the bursty nature of traffic. The curve of BS downturns slightly after network load of 1 and reaches to its $R g$ value at network load of $\sim 1.6$ (when all users are continuously sending at their peak rates, and there is no off time in between). Similarly, throughput curve saturates later for BU and can never reach a value more than $R g$ as of its moderate priority, and it saturates at last for RU at network load of $\sim 1.6$. It implies that the network always has surplus BW (up to network load of $\sim 1.6$ ).

In case of protection, traffic from BS of the failed network (at the network load of 1 which is equivalent to $\sim 1.8 \mathrm{Gbps}$ ) waits in the separate queue of the BS of the network. Intuitively, the network can pass this protected traffic if it has surplus BW, which is always the case as concluded above. For our case study, the throughput of the BS of the failed network starts decreasing sharply (from 100\% to 20\%) for network load of 0.5 to 1 and further decreases at a lower rate (from 20\% to 0\%) for network load of 1 to 1.6 as shown in Fig. $2 \mathrm{~b}$. The cause of the sharp decrease in throughput is increasing $R g$ requirements from lower priority users at higher network loads, and the further decrease is due to the availability of small surplus BW (till 1.6 network load). There is maximum 5-6\% decrease in throughput (for BS after network load of 0.5 because protection traffic is participating in surplus BW division, 2-3\% for BU and RU) as DBA is successfully assigning at least $R g$ to the users of the network whenever there is demand, independent of the network load or protection traffic.

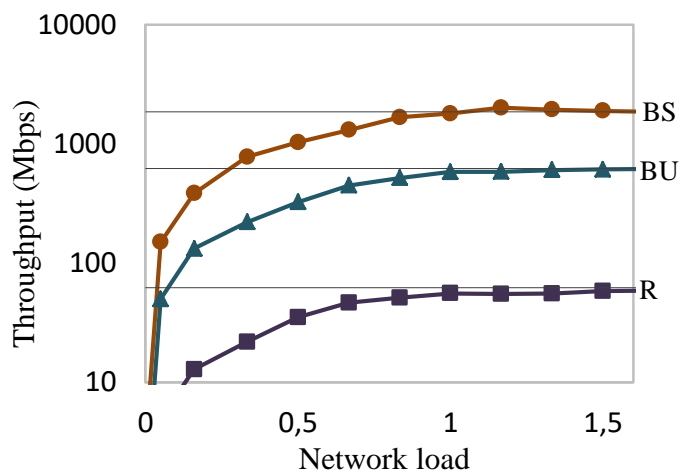

a)

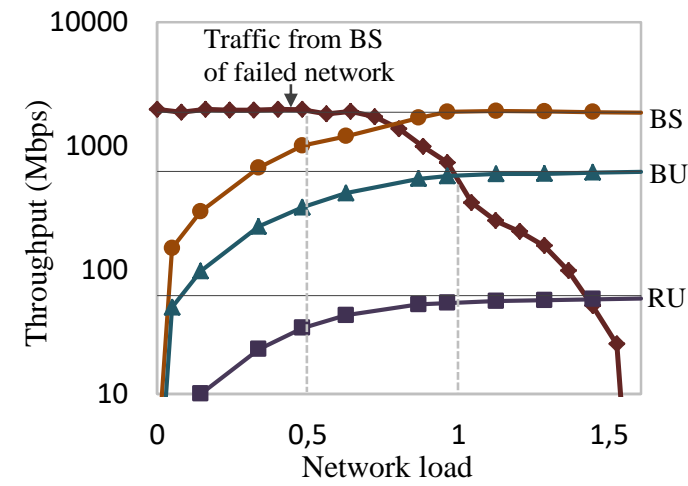

b)

Fig. 2. a) Represents the normal working condition of the network. Throughput saturates earliest for base station (BS), then for the business user $(B U)$, and then for the residential user $(R U)$ because of their assigned priorities. The horizontal lines represent guaranteed BWs of respective users. $\boldsymbol{b})$ The throughput of the BS of the failed network with no guaranteed $B W$ assigned to protected traffic. Throughput of the users of the network experience maximum reduction of 5-6\% after network load of 0.5 .

\subsection{Average packet delay}

Average packet delay is the average delay experienced by a packet from the point of its reception at the queue to the point it reaches OLT. It is usually the sum of queueing delay (waiting time of the packet in the queue), transmission delay (time delay introduced between transmission of first and last bit of packet), and propagation delay (delay experienced to reach OLT from ONU at optical fiber) and averaged among all transmitted packets. 
Average packet delays for the normal working of the network are still higher than the homogeneous case (Fig. 1) since priority is causing higher queue waiting times for RU and BU. In protection case, delay performance of the BSs of the network experiences the highest impact since protection traffic is taking a part of their share. The delay performance is within limits for the normal working condition of the network at around 0.6 of network load ( 6 Gbps). With protection traffic, this factor reduces to 0.4 of network load ( 4 Gbps) which is quite explainable as the net load is again $\sim 6$ Gbps when we add protection traffic (Fig. 3).

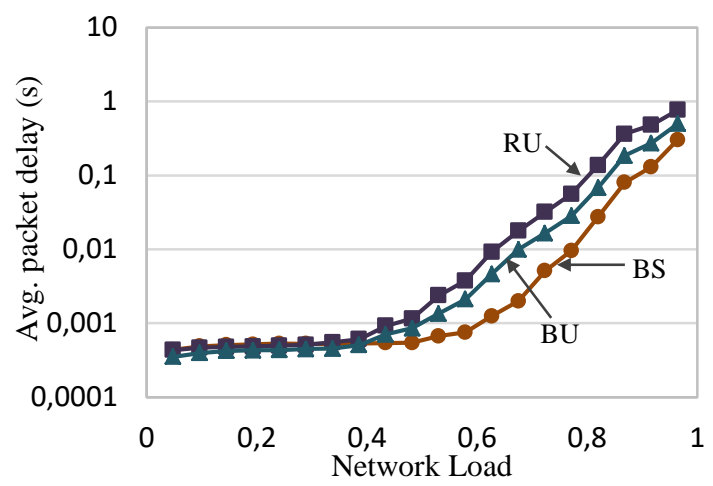

a)

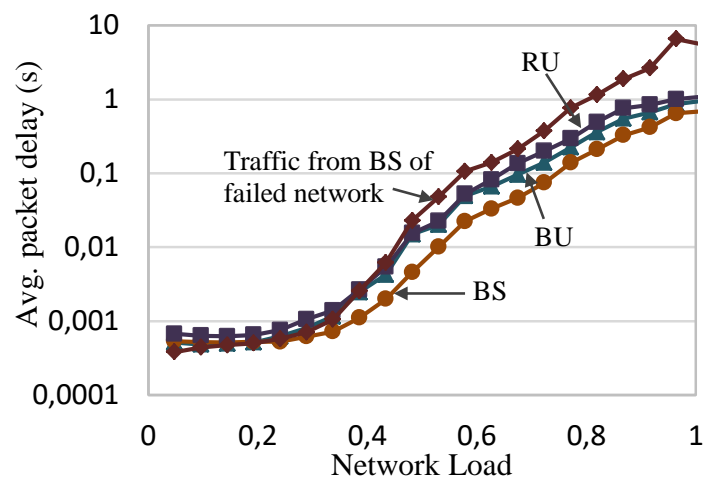

b)

Fig. 3. a) Represents normal working condition of the network with higher priority traffic (of BS) has better delay performance, $\boldsymbol{b})$ Protection case with network traffic from failed node is at network load of 1 ( 1.8 Gbps).

\section{CONCLUSIONS}

XGPON is offering up to 10 Gbps aggregated capacity using TDMA. Heterogeneous users can access to this capacity according to the DBA. In this paper, a DBA has been presented, which considers $D B R u$ for informing newly arrived packets instead of whole buffer occupancy value. Also, it must be kept in mind that we are performing our analysis for worst-case scenarios, while heterogeneity is quite useful as heterogeneous users will never be active simultaneously and in practical systems we can expect better performance.

The user heterogeneity also encourages operators to foresee protection mechanisms when required (e.g., for BS). In this case, the impact of coping with protected traffic from another failed network has been evaluated. It has been shown that in that case, traffic can be protected at the expense of an increase of delay but still below the delay limits of the system. Other alternatives to cope with protected traffic such as other traffic classes definitions, are left for further work.

\section{ACKNOWLEDGMENTS}

This work is performed under the collaboration of Technical University, Munich, and Indian Institute of Technology, Delhi by German Academic Exchange Service (DAAD) India IIT Master Sandwich Programme (IIT), 2017 (57375620).

\section{REFERENCES}

[1] ITU-T Recs., G.984: Gigabit-capable passive optical networks (G-PON), 2003.

[2] ITU-T Recs., G.987: 10-Gigabit-capable passive optical networks (XG-PON), 2010.

[3] A. Shahid and C. Mas Machuca: Dimensioning and Assessment of Protected Converged Optical Access Networks, IEEE Commun. Mag., vol. 55, no. 8, 2017.

[4] ITU-T Rec. G.987.3: 10-Gigabit-capable passive optical networks (XG-PON): TC specifications, 2010.

[5] H. C. Leligou et al.: Efficient medium arbitration of FSAN-compliant GPONs, Int. J. Commun. Syst., vol. 19, no. 5, pp. 603-617, Jun. 2006

[6] M.-S. Han et al.: Efficient dynamic bandwidth allocation for FSAN-Compliant GPON, OSA J. Opt. Netw., vol. 7, no. 8, pp. 783-795, 2008

[7] M. S. Han, H. Yoo, and D. S. Lee: Development of Efficient Bandwidth Allocation Algorithm for XGPON, ETRI J., vol. 35, no. 1, pp. 18-26. 2013.

[8] R. A. Butt, S. M. Idrus, and K. N. Qureshi: Improved dynamic bandwidth allocation algorithm for XGPON, J. Opt Commun Netw., vol. 9, no. 1, pp. 87-97, 2017.

[9] Merayo et al., Fair bandwidth allocation algorithm for PONs based on network utility maximization, J. Opt. Commun. Netw., vol. 9, no. 1, pp. 75-86, 2017.

[10] A. Dixit et al.: Synergized-adaptive multi-GATE polling with void filling: overcoming performance degradation in LR-PONs, J. Opt. Commun. Netw., vol.7, no. 9, pp 837-850, 2015.

[11] ITU-T Recs., G.9807.1: 10-Gigabit-capable symmetric passive optical network (XGS-PON), 2016.

[12] G. Kramer, B. Mukherjee, and G. Pesavento: IPACT: a dynamic protocol for an Ethernet PON (EPON), IEEE Commun. Mag., vol. 40, no. 2, pp. 74-80, 2002.

[13] D. Bertsekas and R. Gallager: Data Networks, NJ, Englewood Cliffs: Prentice-Hall, vol. 2, 1992. 